\title{
ESTABILIZAÇÕES LOMBARES DINÂMICAS
}

\author{
LUMBAR DYNAMIC STABILIZATIONS \\ ESTABILIZACIONES LUMBARES DINÁMICAS
}

Ivan Dias Rocha ${ }^{1}$, Alexandre Fogaça Cristante², Raphael Martus Marcon ${ }^{1}$

\section{RESUMO}

O funcionamento fisiológico normal da coluna depende da movimentação normal de cada unidade motora, que consiste em duas vértebras e o disco intervertebral interposto entre elas. Embora a artrodese da coluna vertebral venha sendo utilizada para o tratamento de diversas doenças da coluna, essa modalidade de tratamento acarreta a perda de movimentação dos níveis em que houve a fusão e como consequência pode sobrecarregar os níveis adjacentes podendo provocar a sua degeneração precoce. Proponentes das técnicas de estabilização dinâmicas acreditam que estas podem levar a correção dos problemas minimizando o risco de degeneração dos níveis adjacentes. Atualmente existem no mercado diversos métodos de estabilização dinâmica anteriores e posteriores. Já existem trabalhos biomecânicos que comprovam o benefício teórico de quase todos eles, porém ainda hoje, faltam ensaios clínicos que comprovem a sua utilidade e segurança por longos períodos de seguimento para o paciente. Portanto é fundamental que estes materiais sejam analisados de maneira acadêmica para que no futuro próximo possam ser utilizados em situações precisas e com segurança para os pacientes.

Descritores: Dor lombar; Artrodese; Coluna vertebral; Estenose espinal.

\begin{abstract}
The normal physiological functioning of the spine depends on the normal movement of each motor unit, which consists of two vertebrae and the intervertebral disc interposed between them. Although fusion of a spine motor unit may be used to treat various diseases of the spine, this modality of treatment results in loss of movement of the levels that were fused and consequently may overload the adjacent levels and may cause its degeneration. Proponents of dynamic stabilization techniques believe that it may lead to correction of problems minimizing the risk of degeneration of adjacent levels. Currently there are different market dynamic stabilization methods. Already there are biomechanical studies that prove the theoretical benefit of almost all of them, but it is still missing clinical trials to prove its usefulness and safety for long periods of follow-up to the patient. It is therefore essential that these materials are considered for prospective clinical trial with long period of follow up so that in the near future they may be used precisely and safely to patients.
\end{abstract}

Keywords: Low back pain; Arthrodesis; Spine; Spinal stenosis.

\section{RESUMEN}

El funcionamiento fisiológico normal de la columna depende de los movimientos normales de cada unidad motora, la cual consiste de dos vértebras y del disco intervertebral interpuesto entre ellas. Aunque la artrodesis de la columna vertebral está siendo utilizada para el tratamiento de diversas enfermedades de la columna, esa modalidad de tratamiento causa la pérdida de los movimientos en los niveles en los que hubo la fusión y, como consecuencia, puede sobrecargar los niveles adyacentes, pudiendo provocar su degeneración precoz. Los proponentes de las técnicas de estabilización dinámica creen que éstas pueden llevar a la corrección de los problemas, minimizando el riesgo de degeneración de los niveles adyacentes. Actualmente, existen en el mercado diversos métodos de estabilización dinámica anterior y posterior. Ya hay trabajos biomecánicos que comprueban el beneficio teórico de casi todos ellos, no obstante, aún en la actualidad, faltan ensayos clínicos que comprueben su utilidad y seguridad durante largos períodos de seguimiento del paciente. Por consiguiente, es fundamental que estos materiales sean analizados, con profundidad universitaria, para que en el futuro próximo puedan ser utilizados en situaciones especificas y con seguridad para los pacientes.

Descriptores: Dolor lumbar; Artrodesis; Columna vertebral; Estenosis espinal.

\section{INTRODUÇÃO}

O funcionamento fisiológico normal da coluna depende da movimentação normal de cada unidade motora, que consiste em duas vértebras e o disco intervertebral interposto entre elas. Embora a artrodese da coluna vertebral venha sendo utilizada para o tratamento de diversas doenças da coluna, essa modalidade de tratamento acarreta a perda de movimentação dos níveis em que houve a fusão e como conseqüência pode sobrecarregar os níveis adjacentes podendo provocar a sua degeneração precoce ${ }^{1-3}$. Proponentes das técnicas de estabilização dinâmicas acreditam que estas podem levar a correção dos problemas minimizando o risco de degeneração dos níveis adjacentes.
Os dispositivos de estabilização dinâmica lombares podem ser anteriores e posteriores. Os anteriores são a prótese de disco e os substitutos do núcleo pulposo. Já os posteriores podem ser divididos em duas categorias: os espaçadores interespinhosos, os estabilizadores pediculares.

Este capítulo será focado nos espaçadores interespinhosos e nos estabilizadores pediculares.

\section{Avaliação Clínica}

A avaliação do paciente candidato a este tipo de procedimento cirúrgico inicia-se com história e exame físico detalhado.

Na história clinica deve-se atentar ao local da dor do paciente,

1. Médico Assistente do Grupo de Coluna do Instituto de Ortopedia e Traumatologia do HC/FMUSP - São Paulo, SP, Brasil.

2. Professor Doutor da Faculdade de Medicina da Universidade de São Paulo, Médico Assistente do Grupo de Coluna do Instituto de Ortopedia eTraumatologia do HC/FMUSP - São Paulo, SP, Brasil.

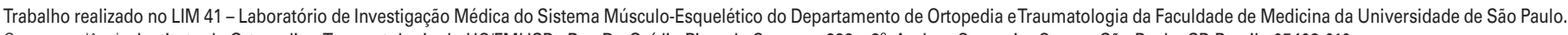
Correspondência: Instituto de Ortopedia eTraumatologia do HC/FMUSP - Rua Dr. Ovídio Pires de Campos, 333 - $3^{\circ}$. Andar - Cerqueira Cesar - São Paulo, SP, Brasil - 05403-010

E-mail: aacristante@uol.com.br 
se há presença de dor irradiada ou referida, se há presença de sintomas de claudicação neurogênica, o tempo e a intensidade da dor do paciente, se há doenças reumatológicas presentes, se houve cirurgia prévia, deve se saber quais foram os tratamentos prévios para sua queixa e por quanto tempo foi feito o tratamento conservador.

No exame físico, deve-se avaliar amplitude de movimento da coluna, pontos de dor local e irradiada, deve-se observar se há Laségue positivo, deve avaliar as articulações sacro ilíacas, os quadris e os joelhos a procura de lesões locais e deve-se realizar um exame neurológico minucioso.

\section{Avaliação Radiológica}

O paciente deve ser avaliado com radiografias em AP, Perfil e perfil dinâmico a procura de deformidades, listeses, e instabilidades locais. Nos pacientes com listese de alto grau, seria uma contra-indicação para os procedimentos de estabilidade dinâmica.

O paciente ainda deve ser submetido à Ressonância Nuclear Magnética da coluna lombo-sacra para avaliar as possíveis causas da sua dor. Entre as causas principais destacam-se a doença degenerativa discal, a hérnia de disco, a estenose do canal veertebral e a artrose facetária.

\section{Espaçadores Interespinhosos}

O conceito dos espaçadores interespinhosos, iniciou-se com a observação que muitos pacientes com sintomas de estenose do canal vertebral pioram com a extensão e melhoram com a flexão do segmento vertebral acometido ${ }^{4}$. Os espaçadores interespinhosos, biomecanicamente, atuam, em geral, no sentido de impedir a extensão da unidade funcional, diminuído a carga em cima das facetas, e aumentando a área interna do canal vertebral, sem no entanto interferir com a flexão ou a lateralização da coluna ${ }^{5-7}$. O aumento da área interna do canal vertebral acontece principalmente, devido a diminuição da redundância do ligamento amarelo que está presente nestes pacientes e devido a diminuição da pressão discal posterior, diminuindo assim o abaulamento discal. Existem ainda estudos que comprovam que este tipo de dispositivo não altera a pressão dos discos dos segmentos adjacentes assim como não altera a movimentação dos mesmos ${ }^{7}$.

O primeiro trabalho com espaçadores espinhosos foi feito com o chamado "Minns" que era um dispositivo de silicone em forma de halteres. O estudo "in vitro" provou que o espaçador era capaz de diminuir a pressão no disco e nas facetas no segmento em que foi implantado, porém não houve ensaios clínicos com este material ${ }^{8}$.

Existem atualmente alguns dispositivos no mercado entre eles podemos citar o DIAM (Meditronic), o Wallis (Abbott) e o X-Stop (Zimmer).

O DIAM da Meditronic consiste em um espaçador de silicone coberto com polietileno. Ele é seguro no local de implantação por duas fitas, uma colocado no espinhoso acima e outra abaixo (Figura 1). Schiavone e Pasquale em seu estudo com este material, realizaram uma série de casos com 22 pacientes e seguimento de 10 meses. Neste estudo o autor descreve que 16 pacientes apresentaram melhora excelente e quatro tiveram boa melhora. $\mathrm{O}$ autor conclui o material é simples e de fácil implantação, porém necessita de trabalhos controlados randomizados para melhor avaliação9 .

O segundo implante denominado Wallis da Abbott (Figura 2) foi desenvolvido por Senegas. O Implante é composto por um espaçador de PEEK (polietereterquetone) e duas fitas que são passadas no processo espinhoso superior e no inferior. Segundo o autor as indicações para o método não se resume a estenose de canal e pode ser utilizado nas seguintes situações: pós discectomia em que foi retirada grande quantidade de disco, em uma nova discectomia por recidiva de hérnia de disco, em uma discectomia em um nível de transição como L5 sacralizado, em caso de lombalgia com alteração Modic tipo 1 e na doença degenerativa discal de nível adjacente a uma fusão ${ }^{10}$. No seu estudo mais recente Senegas, em sua série de casos avaliou 107 pacientes com implantação de Wallis em pacientes com hérnia de disco e estenose do canal com seguimento de 13 anos. Foi necessário a retirada do material em 20 pacientes que foram submetidos à fusão, os demais 87 pacientes, mantiveram o material pelo período de estudo e apresentavam melhora dos índices SF-36, Owestry e VAS significantes $(p<0,05)^{11}$

O último implante, denominado X-Stop, (Figura 3) por sua vez é um implante de titânio, e não possui nenhum tipo de fita para manutenção de sua posição. Fundamentalmente é usado em casos de estenose de canal associados a sintomas de claudicação neurogênica ${ }^{12}$. A técnica de implantação é simples e rápida, podendo até em alguns casos ser feita sob anestesia local apenas. Zucherman et al..$^{13} \mathrm{em}$ um estudo randomizado multicêntrico com 191 pacientes e seguimento de dois anos encontrou melhores resultados com o uso do X-Stop do que com uso de infiltração epidural (controle).

Devido às características anatômicas, nenhum dos implantes interespinhosos é adequado para ser utilizado no espaço L5-S1.

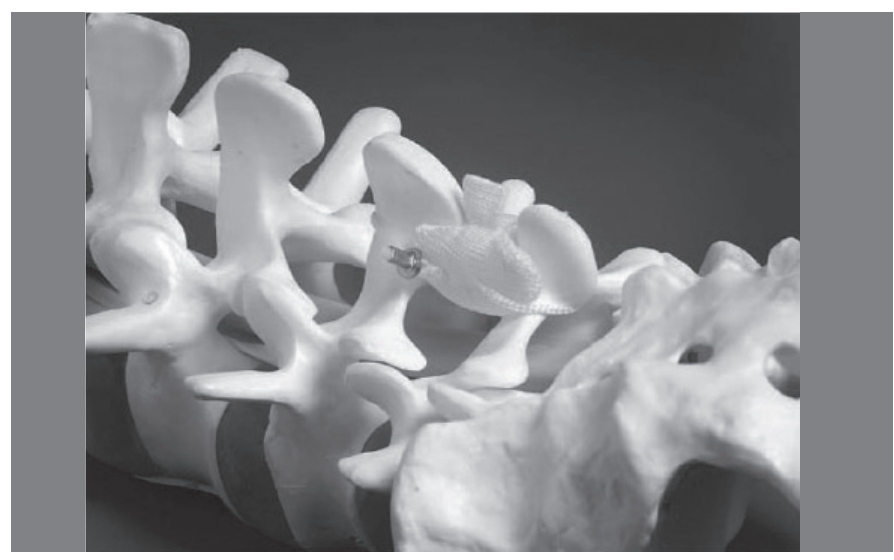

Figura 1. llustração do espaçador interespinhoso DIAM.

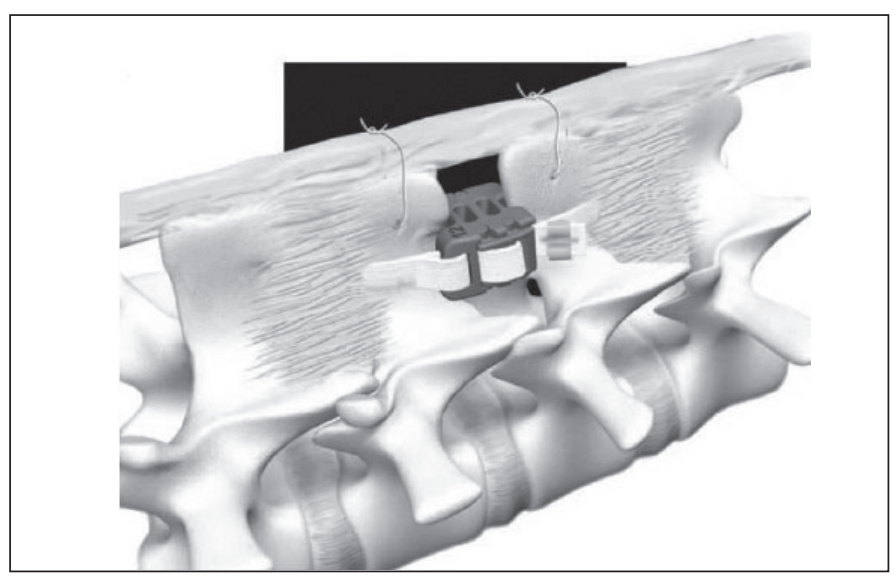

Figura 2. Ilustração do espaçador interespinhoso WALLIS.

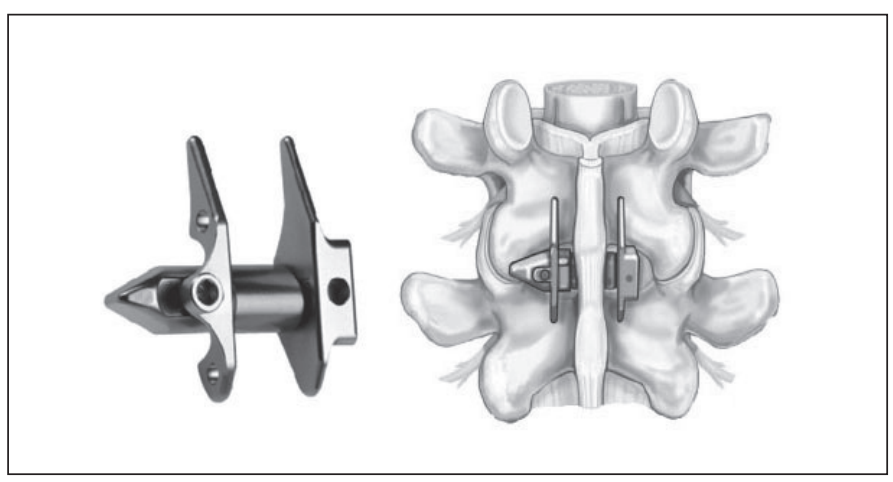

Figura 3. llustração do espaçador interespinhoso XTOP. 


\section{Estabilizadores Pediculares}

Nas últimas duas décadas a principal justificativa para a fusão do segmento motor foi o conceito de instabilidade. Embora ainda hoje este conceito seja difícil de definir, pacientes que apresentam segmentos com mobilidade anormal nas radiografias em flexo-extensão ${ }^{14}$, especialmente nos casos de espondilolisteses, se beneficiariam da fusão ${ }^{15}$. Porém a grande maioria dos pacientes com lombalgia não apresenta qualquer sinal de instabilidade ${ }^{16}$.

Atualmente acredita-se que os sintomas de dor lombar em geral estão mais associados com a descarga anormal de peso do que alteração da movimentação ${ }^{17}$

A dor relacionada ao segmento motor pode originar: das placas terminais vertebrais, do anulo discal, do periósteo vertebral, das facetas e até dos tecidos moles ao redor destas estruturas ${ }^{18}$.

As alterações degenerativas presentes no disco parecem iniciar o processo e ocorrem de maneira irregular, alterando as propriedades isotrópicas do mesmo. Assim o disco se torna não homogêneo, com áreas de colágeno condensado e áreas de colágeno degenerado. A distribuição das cargas nas placas terminais se torna assim não uniforme, com áreas de maior pressão e de menor pressão ${ }^{17}$.

A má distribuição das cargas pode se intensificar ainda com a posição do paciente, piorando ainda mais a distribuição das pressões (teoria da pedra no sapato) ${ }^{17}$

Com o tempo e a progressão da degeneração, o disco se torna mais homogêneo e com menos movimento, o que em grande parte dos pacientes acarreta da melhora da dor ${ }^{18}$

Essa teoria ajuda a explicar alguns dilemas que ainda estão presentes a respeito da dor lombar, como porque paciente pode apresentar degeneração discal em exames de imagem sem dor e porque a lombalgia tem um componente postural importante.

Os estabilizadores pediculares diminuem o movimento patológico anormal mantendo a movimentação e melhorando a distribuição das cargas no disco, podendo ser usados em qualquer segmento lombar ${ }^{18}$.

Os dispositivos pediculares dinâmicos tiveram seu início com Graf que inventou o sistema ligamentar de $\mathrm{Graf}^{19}$ (Figura 4). Este sistema consistia em uma banda não elástica ao redor de dois parafusos, a coluna seria colocada em leve extensão e a banda então era colocada. O dispositivo foi desenvolvido para anular as forcas rotacionais da coluna travando as facetas em extensão. Este sistema permitia movimentação em flexão e tinha como o grande problema o fato de causar uma estenose foraminal. Apesar de ter sido amplamente utilizado não há nenhum estudo de boa qualidade a respeito deste material e estudos recentes como o de Rigby et al. ${ }^{20}$ desaconselharam o uso deste sistema.

O segundo sistema denominado Dynesis (Zimmer) (Figura 5) tem uma proposta diferente, pois tenta controlar tanto a flexão como a extensão. Ele consiste em uma banda de tensão com um espaçador elástico. Em flexão há um cordão que atua com banda de tensão controlando este movimento e em extensão os tubos elásticos atuam como um espaçador elástico restringindo a amplitude deste movimento ${ }^{16}$.

Schnake et al. ${ }^{21}$ avaliaram em uma série de casos com 26 pacientes com diagnostico de estenose de canal e claudicação neurogênica a técnica de descompressão associada a estabilização dinâmica com Dynesis. Em seu estudo obteve $87,5 \%$ de sucesso terapêutico, porém teve 17\% de soltura do material. Segundo o autor nos pacientes em que houve soltura do material, não foi necessário uma reabordagem, pois os pacientes não eram sintomáticos.

Stoll et al. ${ }^{22}$ realizaram um estudo prospectivo multicêntrico para avaliar a eficácia e a segurança do Dynesis no tratamento de diversas patologias da coluna lombar. Foram avaliados e operados 83 pacientes com as seguintes patologias: (60.2\%) estenose de
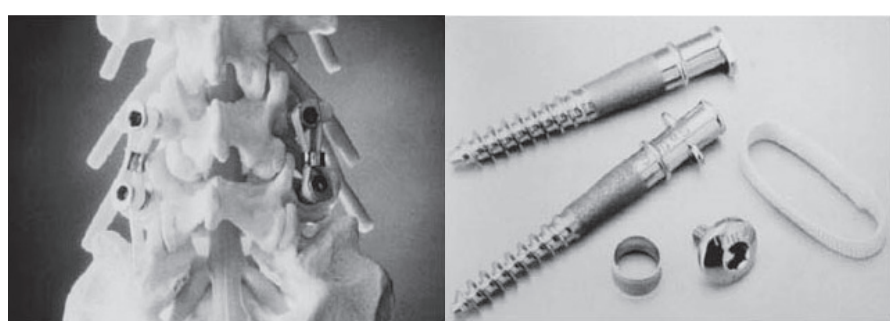

Figura 4. llustração do sistema de fixação dinâmica baseado em pedículo de GRAF. canal, $(24,1 \%)$ degeneração discal, $(8,4 \%)$ herniação de disco e (6\%) revisão de cirurgia de coluna. O seguimento foi de 38 meses. Esse estudo mostrou resultados de melhora da dor e de taxas de reoperações comparáveis as técnicas de fusão.

Grob et al. ${ }^{23}$ em outro estudo, com 31 casos em 2 anos de seguimento, em que foi avaliado a satisfação do paciente, obteve melhora da dor em $67 \%$ porém $50 \%$ dos pacientes não realizariam novamente este procedimento e teve taxas de $17 \%$ de soltura de material e $19 \%$ de reoperações.

Com relação ao Dynesis podemos concluir que apesar de ter sido demonstrado em estudos em cadáveres uma diminuição na pressão intra-discal e uma melhora na movimentação da unidade motora ${ }^{24}$, ainda não há uma indicação precisa para o método e faltam estudos de qualidade controlados, randomizados que demonstrem um resultado satisfatório para o seu uso.

Existem ainda alguns outros dispositivos que vem sendo usados como o Cosmic (Ulrich-Technicare) e outros que ainda estão em fase de teste como o TOPS (prótese dos elementos posteriores) e o FASS ( Sistema de estabilização com fulcro).

No caso do sistema Cosmic, (Figura 6) não há espaçador de borracha. O sistema consiste de parafusos e hastes rígidas porem na interface entre eles é permitido alguma movimentação apenas no plano sagital ${ }^{25}$.

Já o TOPS material desenvolvido por Wilke et al. ${ }^{26}$ (Figura 7 ) o conceito é a substituição de todos os elementos posteriores inclusive

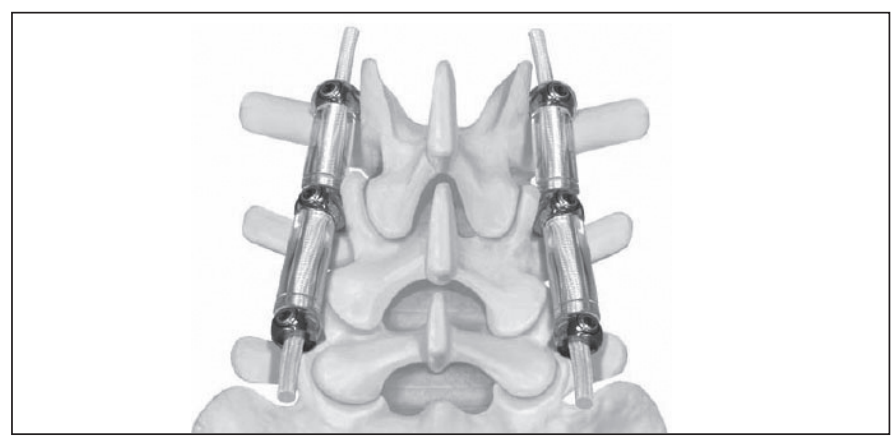

Figura 5. llustração do sistema de fixação dinâmica baseado em pedículo DYNESIS.

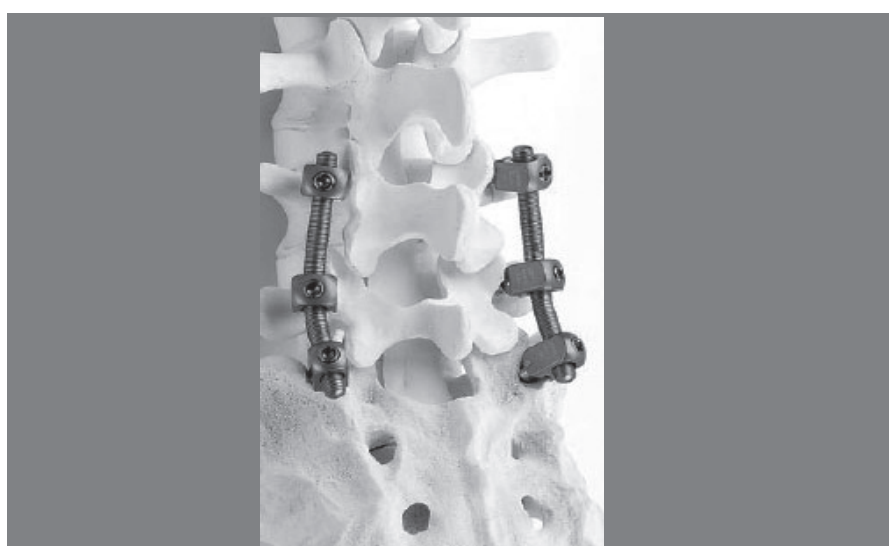

Figura 6. Ilustração do sistema de fixação dinâmica baseado em pedículo COSMIC.

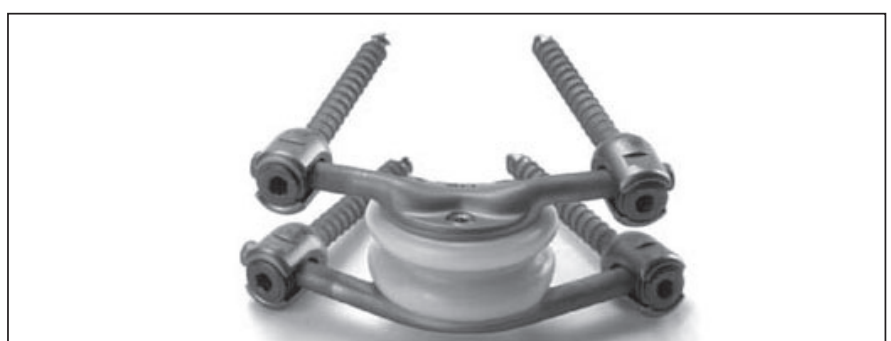

Figura 7. Ilustração do sistema TOPS de substituição de todos os elementos posteriores inclusive das facetas. 
das facetas. O implante consiste de parafusos pediculares e uma estrutura central que lembra uma prótese de disco, capaz de distribuir tanto forças de compressão como forças de tração. Este material foi avaliado apenas em estudos biomecânicos com cadáveres em que foi demonstrado que ele consegue mimetizar $85 \%$ do movimento normal da coluna após ampla descompressão.

Recentemente também dois autores, Sengupta e Mulholland²7 descreveram um novo material para estabilização dinâmica, denominado FASS (Figura 8). Este implante consiste de parafusos e uma banda de tensão posterior semelhante à técnica de Graf, porém aqui há um fulcro bem próximo a inserção dos parafusos (Figura 9). O estudo realizado em cadáveres demonstrou que com a inserção do fulcro, há uma melhor redução da pressão discal, principalmente na parte posterior, sem causar cifose no seguimento motor, o que seria o principal problema biomecânico do Dynesis²7, este material não esta disponível no mercado.

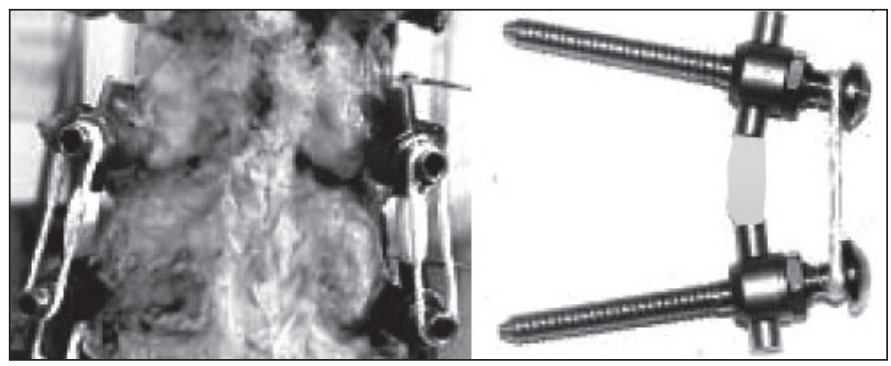

Figura 8. llustração do sistema de estabilização dinâmica baseado em pedículo FASS.
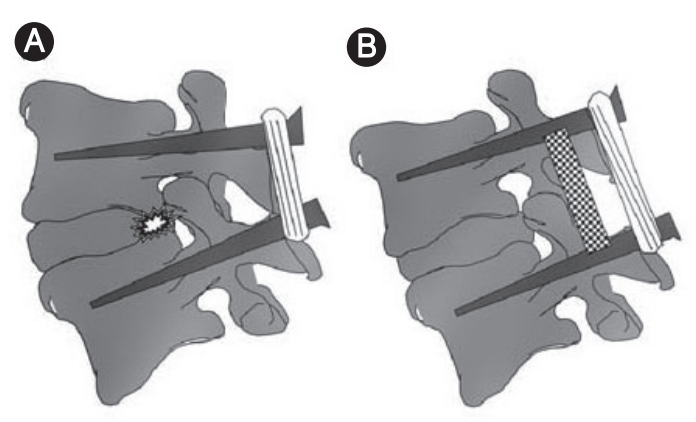

Figura 9. Ilustração comparando os sistema de fixação dinâmica GRAF e FASS.

\section{CONCLUSÃO}

Atualmente existem no mercado diversos métodos de estabilização dinâmica anteriores e posteriores. Já existem trabalhos biomecânicos que comprovam o benefício teórico de quase todos eles, porém ainda hoje, faltam ensaios clínicos que comprovem a sua utilidade e segurança por longos períodos de seguimento para o paciente. Portanto é fundamental que estes materiais sejam analisados de maneira acadêmica para que no futuro próximo possam ser utilizados em situações precisas e com segurança para os pacientes.

\section{REFERÊNCIAS}

1. Schlegel JD, Smith JA, Schleusener RL. Lumbar motion segment pathology adjacent to thoracolumbar, lumbar, and lumbosacral fusions. Spine (Phila Pa 1976). 1996;21(8):970-81.

2. Aota Y, Kumano K, Hirabayashi S. Postfusion instability at the adjacent segments after rigid pedicle screw fixation for degenerative lumbar spinal disorders. J Spinal Disord. 1995:8(6):464-73

3. Etebar S, Cahill DW. Risk factors for adjacent-segment failure following lumbar fixation with rigid instrumentation for degenerative instability. J Neurosurg. 1999;90(2 Suppl):163-9.

4. Verbiest $H$. A radicular syndrome from developmental narrowing of the lumbar vertebral canal. J Bone Joint Surg Br. 1954;36(2):230-7.

5. Siddiqui M, Nicol M, Karadimas E, Smith F, Wardlaw D. The positional magnetic resonance imaging changes in the lumbar spine following insertion of a novel interspinous process distraction device. Spine (Phila Pa 1976). 2005;30(23):2677-82.

6. Christie SD, Song JK, Fessler RG. Dynamic interspinous process technology. Spine (Phila Pa 1976). 2005;30(16 Suppl):S73-8.

7. Lindsey DP, Swanson KE, Fuchs P, Hsu KY, Zucherman JF, Yerby SA. The effects of an interspinous implant on the kinematics of the instrumented and adjacent levels in the lumbar spine. Spine (Phila Pa 1976). 2003;28(19):2192-7.

8. Minns RJ, Walsh WK. Preliminary design and experimental studies of a novel soft implant for correcting sagittal plane instability in the lumbar spine. Spine (Phila Pa 1976). 1997;22(16):1819-25; discussion 26-7.

9. Schiavone AM, Pasquale G. The use of disc assistance prostheses (Diam) in degenerative lumbar pathology: Indications, technique, and results. Ital J Spinal Disord. 2003;3:213-20

10. Senegas J. Mechanical supplementation by non-rigid fixation in degenerative intervertebral lumbar segments: the Wallis system. Eur Spine J. 2002:11(Suppl 2):S164-9.

11. Senegas J, Vital JM, Pointillart V, Mangione P. Clinical evaluation of a lumbar interspinous dynamic stabilization device (the Wallis system) with a 13-year mean follow-up. Neurosurg Rev. 2009:32(3):335-41; discussion 41-2.

12. Swanson KE, Lindsey DP, Hsu KY, Zucherman JF, Yerby SA. The effects of an interspinous implant on intervertebral disc pressures. Spine (Phila Pa 1976). 2003;28(1):26-32.

13. Zucherman JF, Hsu KY, Hartjen CA, Mehalic TF, Implicito DA, Martin MJ, et al. A multicenter, prospective, randomized trial evaluating the X STOP interspinous process decompression system for the treatment of neurogenic intermittent claudication: two-year follow-up results. Spine (Phila Pa 1976). 2005;30(12):1351-8.
14. Dvorak J, Panjabi MM, Chang DG, Theiler R, Grob D. Functional radiographic diagnosis of the lumbar spine. Flexion-extension and lateral bending. Spine (Phila Pa 1976). 1991;16(5):562-71.

15. Kwon BK, Vaccaro AR, Grauer JN, Beiner J. Indications, techniques, and outcomes of posterior surgery for chronic low back pain. Orthop Clin North Am. 2003:34(2):297-308.

16. Nockels RP. Dynamic stabilization in the surgical management of painful lumbar spinal disorders. Spine (Phila Pa 1976). 2005;30(16 Suppl):S68-72.

17. Sengupta DK. Dynamic stabilization devices in the treatment of low back pain. Orthop Clin North Am. 2004;35(1):43-56.

18. Mulholland RC, Sengupta DK. Rationale, principles and experimental evaluation of the concept of soft stabilization. Eur Spine J. 2002:11(Suppl 2):S198-205.

19. Graf H. Lumbar instability: surgical treatment without fusion. Rachis. 1992(412):123-37.

20. Rigby MC, Selmon GP, Foy MA, Fogg AJ. Graf ligament stabilisation: mid- to long-term follow-up. Eur Spine J. 2001;10(3):234-6.

21. Schnake KJ, Schaeren S, Jeanneret B. Dynamic stabilization in addition to decompression for lumbar spinal stenosis with degenerative spondylolisthesis. Spine (Phila Pa 1976). 2006:31(4):442-9.

22. Stoll TM, Dubois G, Schwarzenbach 0 . The dynamic neutralization system for the spine: a multi-center study of a novel non-fusion system. Eur Spine J. 2002;11 (Suppl 2):S170-8.

23. Grob D, Benini A, Junge A, Mannion AF. Clinical experience with the Dynesys semirigid fixation system for the lumbar spine: surgical and patient-oriented outcome in 50 cases after an average of 2 years. Spine (Phila Pa 1976). 2005;30(3):324-31.

24. Schmoelz W, Huber JF, Nydegger T, Dipl I, Claes L, Wilke HJ. Dynamic stabilization of the lumbar spine and its effects on adjacent segments: an in vitro experiment. J Spinal Disord Tech. 2003;16(4):418-23.

25. Strempel AV, Mosmann D, Stoss C. Stabilisation of the degenerated lumbar spine in the nonfusion technique with cosmic posterior dynamic system. Word Spine J. 2006;1(1):40-7.

26. Wilke HJ, Schmidt H, Werner K, Schmolz W, Drumm J. Biomechanical evaluation of a new total posterior-element replacement system. Spine (Phila Pa 1976). 2006;31(24):2790-6.

27. Sengupta DK, Mulholland RC. Fulcrum assisted soft stabilization system: a new concept in the surgical treatment of degenerative low back pain. Spine (Phila Pa 1976). 2005;30(9):1019-29. 